\title{
Collective States of Interacting Fibonacci Anyons
}

\author{
Simon Trebst, ${ }^{1}$ Eddy Ardonne, ${ }^{2,3}$ Adrian Feiguin, ${ }^{1}$ David A. Huse, ${ }^{4}$ Andreas W. W. Ludwig, ${ }^{5}$ and Matthias Troyer $^{6}$ \\ ${ }^{1}$ Microsoft Research, Station Q, University of California, Santa Barbara, California 93106, USA \\ ${ }^{2}$ California Institute of Technology, Pasadena, California 91125, USA \\ ${ }^{3}$ Nordita, Roslagstullsbacken 23, SE-106 91 Stockholm, Sweden \\ ${ }^{4}$ Department of Physics, Princeton University, Princeton, New Jersey 08544, USA \\ ${ }^{5}$ Physics Department, University of California, Santa Barbara, California 93106, USA \\ ${ }^{6}$ Theoretische Physik, Eidgenössische Technische Hochschule Zürich, 8093 Zürich, Switzerland
}

(Received 4 February 2008; published 30 July 2008)

\begin{abstract}
We show that chains of interacting Fibonacci anyons can support a wide variety of collective ground states ranging from extended critical, gapless phases to gapped phases with ground-state degeneracy and quasiparticle excitations. In particular, we generalize the Majumdar-Ghosh Hamiltonian to anyonic degrees of freedom by extending recently studied pairwise anyonic interactions to three-anyon exchanges. The energetic competition between two- and three-anyon interactions leads to a rich phase diagram that harbors multiple critical and gapped phases. For the critical phases and their higher symmetry end points we numerically establish descriptions in terms of two-dimensional conformal field theories. A topological symmetry protects the critical phases and determines the nature of gapped phases.
\end{abstract}

DOI: 10.1103/PhysRevLett.101.050401

PACS numbers: 05.30.Pr, 03.65.Vf, 73.43.Lp

Two-dimensional topological quantum liquids such as the fractional quantum Hall $(\mathrm{FQH})$ states harbor exotic quasiparticle excitations which due to their unusual exchange statistics are referred to as anyons. Interchanging two anyons may result in not only a fractional exchange phase, but may also give rise to a unitary rotation of the original wave function in a degenerate ground-state manifold. This latter case of non-Abelian statistics is proposed to be exploited in the context of topological quantum computation [1,2]. Intense experimental efforts [3-5] are currently under way to demonstrate the non-Abelian character of quasiparticle excitations in certain $\mathrm{FQH}$ states, as proposed theoretically $[6,7]$.

Given a set of several non-Abelian anyons we can ask what kind of collective states are formed if these anyons are interacting with each other. A first step in this direction has recently been taken by studying chains of "Fibonacci anyons" with nearest-neighbor interactions [8]. Fibonacci anyons represent the non-Abelian part of the quasiparticle statistics in the $k=3 Z_{k}$-parafermion "Read-Rezayi" state [6], an effective theory for FQH liquids at filling fraction $\nu=12 / 5$ [9]. A single Fibonacci anyon carries a topological charge $\tau$. Two such anyons may combine ("fuse") so the pair has charge $\tau$ or has no charge, which is denoted 1. This is analogous to two $S U(2)$ spin-1/2's combining to either spin-1 or spin-zero total spin. A two-anyon interaction assigns different energy to the two possible charges of the pair, just as a Heisenberg exchange interaction does for the two possible total values of spin of a pair of $S U(2)$ spin-1/2's. For a chain of Fibonacci anyons with a uniform pairwise nearest-neighbor interaction of either sign it has been explicitly shown [8] that the Hamiltonian has a topological symmetry, which was predicted to stabilize one of the gapless phases. In this Letter, we give a broader per- spective on possible collective phases of interacting Fibonacci anyons and phase transitions between them.

The specific model we will focus on has, in addition to the two-anyon term, an additional three-anyon interaction both of which may arise from tunneling [10]. We find a rich ground-state phase diagram that harbors multiple critical, gapless, and gapped phases. The topological symmetry, introduced in Ref. [8], that measures the topological flux through a ring of Fibonacci anyons plays an essential role in determining the nature of the observed phases and phase transitions. In particular, we find that this topological symmetry protects all the critical phases against spatially uniform local perturbations. These extended critical phases can be described in terms of 2D conformal field theories (CFT) with central charges $c=7 / 10$ and $c=4 / 5$ and can be, respectively, mapped exactly onto the tricritical Ising and 3-state Potts critical points of the generalized hard hexagon model $[8,11]$. At the phase transitions out of the tricritical Ising phase into adjacent gapped phases the system exhibits even higher symmetries which we identify as tetracritical Ising and 3-state Potts critical points. This demonstrates that these 2D classical models share an identical nonlocal symmetry which is the classical analog of the topological symmetry in the 1D quantum chains. At the transition into the gapped phases this topological symmetry is spontaneously broken which results in a nontrivial ground-state degeneracy in the gapped phases.

Two-anyon interactions. -For a uniform chain of Fibonacci anyons the Hamiltonian introduced in Ref. [8] energetically favors one or the other of the possible fusion products of two neighboring $\tau$-particles which, by the fusion rule $\tau \times \tau=1+\tau$, can be either a 1 or a $\tau$. The energy of the former is lower for a coupling that is termed "antiferromagnetic" (AFM), in analogy to the familiar 
$S U(2)$ spins-chains, while that of the latter is lower with a coupling termed "ferromagnetic" (FM). The underlying Hilbert space is spanned by an orthonormal basis of states, each state corresponding to one possible labeling of the chain [8] of repeated fusions with $\tau$. Each site along this chain of fusions has either a 1 or a $\tau$, with a constraint forbidding two adjacent 1's.

By performing a sequence of local basis transformations and projection onto one of the two fusion channels for each pair of neighboring anyons, the resulting two-anyon interaction Hamiltonian can be written as a sum of local 3-site operators $H=J_{2} \sum_{i} H_{2}^{i}$ which take the explicit form ( $i$ denotes the first in a triple of adjacent sites)

$$
\begin{aligned}
H_{2}^{i}= & -\mathcal{P}_{1 \tau 1}-\phi^{-2} \mathcal{P}_{\tau 1 \tau}-\phi^{-1} \mathcal{P}_{\tau \tau \tau} \\
& -\phi^{-3 / 2}(|\tau 1 \tau\rangle\langle\tau \tau \tau|+\text { H.c. }),
\end{aligned}
$$

where $\mathcal{P}_{a}$ projects onto the state $|a\rangle$, e.g. $\mathcal{P}_{1 \tau 1}=|1 \tau 1\rangle \times$ $\langle 1 \tau 1|$ and $\phi=(1+\sqrt{5}) / 2$ is the golden ratio [8].

Here we want to explore a larger space of models than that given by this uniform chain with only nearest-neighbor two-anyon interactions. One way is to let the strength $J_{2}$ of the interaction alternate along the chain, as illustrated in Fig. 1(a). Two chains can be coupled to form a two-leg ladder. Another way is to add a spatially-uniform threeanyon interaction, as indicated in Fig. 1(b), which because of its rich phase diagram, we discuss in the following.

Majumdar-Ghosh chain. - Three $S U(2)$ spin-1/2's can combine to a total spin $3 / 2$ or $1 / 2$. For a uniform $S U(2)$ spin- $1 / 2$ chain, Majumdar and Ghosh (MG) showed that an AFM coupling (favoring total spin 1/2) for each set of three neighboring spins gives rise to a gapped phase with the two possible dimer coverings being the exact ground states [12]. In the same spirit we have asked what possible phases can be stabilized by a spatially uniform threeparticle interaction term in our anyonic generalization of the $S U(2)$ Heisenberg model, i.e., a term that energetically favors each set of three adjacent Fibonacci anyons to fuse together [10] into either a 1 or a $\tau$, as illustrated in Fig. 1(b). Like the pairwise interaction term such a threeparticle interaction term respects both the translational and topological symmetries. We find that the energetic competition between such two- and three-anyon interactions gives rise to the rich ground-state phase diagram shown in Fig. 2, which we discuss in some detail in the following. Similar to the derivation for the pairwise interaction term (1) we can obtain a local form $H=J_{3} \sum_{i} H_{3}^{i}$ of the threeanyon interaction term by a sequence of basis transforma- a)

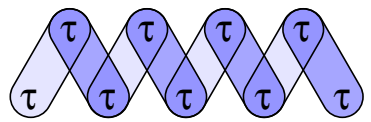

b)

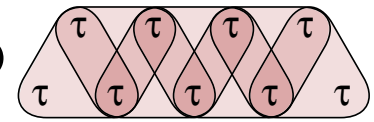

FIG. 1 (color online). Illustration of (a) the alternating chain and (b) the generalized Majumdar-Ghosh chain with a threeanyon interaction term. Shaded enclosures indicate the fusion products that are energetically biased by the Hamiltonian. tions and projections which then takes the explicit form of a 4-site interaction between consecutive labels along the chain of fusions

$$
\begin{aligned}
H_{3}^{i}= & \mathcal{P}_{\tau 1 \tau 1}+\mathcal{P}_{1 \tau 1 \tau}+\mathcal{P}_{\tau \tau \tau 1}+\mathcal{P}_{1 \tau \tau \tau}+2 \phi^{-2} \mathcal{P}_{\tau \tau \tau \tau} \\
& +\phi^{-1}\left(\mathcal{P}_{\tau 1 \tau \tau}+\mathcal{P}_{\tau \tau 1 \tau}\right)-\phi^{-2}(|\tau \tau 1 \tau\rangle\langle\tau 1 \tau \tau|+\text { H.c. }) \\
& +\phi^{-5 / 2}(|\tau 1 \tau \tau\rangle\langle\tau \tau \tau \tau|+| \tau \tau 1 \tau\rangle\langle\tau \tau \tau \tau|+\text { H.c. }), \quad \text { (2) }
\end{aligned}
$$

where the site $i$ denotes the first position in each "quad" of sites. The full Hamiltonian with competing fusion terms then becomes $H_{J_{2}, J_{3}}=\sum_{i}\left(J_{2} H_{2}^{i}+J_{3} H_{3}^{i}\right)$, where we parameterize the couplings by the angle $\theta$ as $J_{2}=\cos \theta$ and $J_{3}=\sin \theta$. We study periodic chains of $L$ anyons.

The phase diagram of this model, shown in Fig. 2, exhibits two critical phases that contain the two exactly solvable points $(\theta=0, \pi)$. These extended critical phases can be described by 2D conformal field theories and are thereby related to $2 \mathrm{D}$ classical critical points to which an exact mapping was established at the two solvable points [8]. For AFM pair interaction $\left(J_{2}>0\right)$ this is the tricritical Ising model $(c=7 / 10)$, while for FM pair interaction $\left(J_{2}<0\right)$ it is the critical point of the 3-state Potts model $(c=4 / 5)$. In particular, we note that the critical phases found at the exactly solvable points are stable upon introducing a small three-anyon fusion term. While the $J_{3}$-term respects both translational and topological symmetries, all translational invariant operators with scaling dimension $<2$ at the exactly solvable points are found to break the

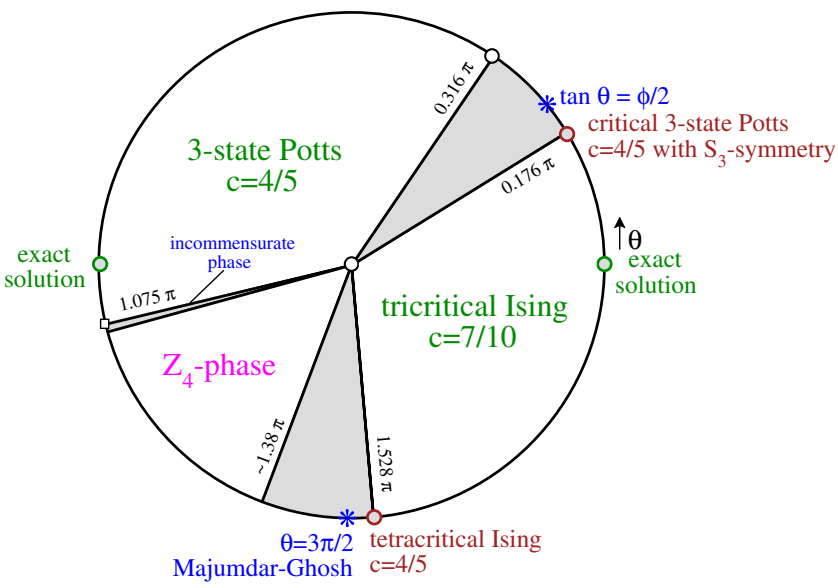

FIG. 2 (color online). The phase diagram of our anyonic Majumdar-Ghosh chain on the circle parameterized by $\theta$, with pairwise fusion term $J_{2}=\cos \theta$ and three-particle fusion term $J_{3}=\sin \theta$. Besides extended critical phases around the exactly solvable points $(\theta=0, \pi)$ that can be mapped to the tricritical Ising model and the 3-state Potts model, there are two gapped phases (gray filled). The phase transitions (red circles) out of the tricritical Ising phase exhibit higher symmetries and are both described by CFTs with central charge 4/5. In the gapped phases exact ground states are known at the positions marked by the stars. In the lower left quadrant a small sliver of an incommensurate phase occurs and a phase which has $Z_{4}$ symmetry. These latter two phases also appear to be critical. 
topological symmetry [8]. This shows that the topological symmetry protects the gaplessness in the vicinity of these points, somewhat analogously to the much-discussed notion that a topological symmetry protects a ground-state degeneracy in a gapped topological phase in 2D space.

For large three-particle interactions these critical phases eventually give way to other phases, such as the two distinct gapped phases indicated by the gray shaded arcs in the phase diagram. Remarkably, the transition to the gapped phase from the tricritical Ising phase when both interaction terms are AFM apparently has an "emergent" $S_{3}$ (3-state Potts) symmetry. Our numerical analysis shows that this transition occurs at $\theta \cong 0.176 \pi$ and is described by the parafermion CFT with central charge $c=4 / 5$, indicative of an additional $S_{3}$-symmetry at this point. Figure 3 shows the rescaled energy spectrum at this critical point whose (universal) low-energy part is in spectacular agreement with the CFT predictions. Note the relevant operator with zero momentum, zero flux, and scaling dimension $4 / 3$, which breaks the $S_{3}$ symmetry. It is the leading operator present in the Hamiltonian away from this special point, and drives the system into either the gapped or the tricritical Ising phase. In the gapped phase, the topological symmetry is spontaneously broken and the resulting ground state, which has zero total momentum, is twofold degenerate in the thermodynamic limit. In the tricritical Ising phase, the $Z_{2}$ sublattice-symmetry breaking order parameter corresponds to a more relevant continuum operator than the topological order parameter, while it is the state corresponding to the $Z_{2}$ order parameter which acquires a higher energy in the gapped phase, where only the topological symmetry is broken. At the transition, both order parameters are degenerate, see Fig. 3, and together

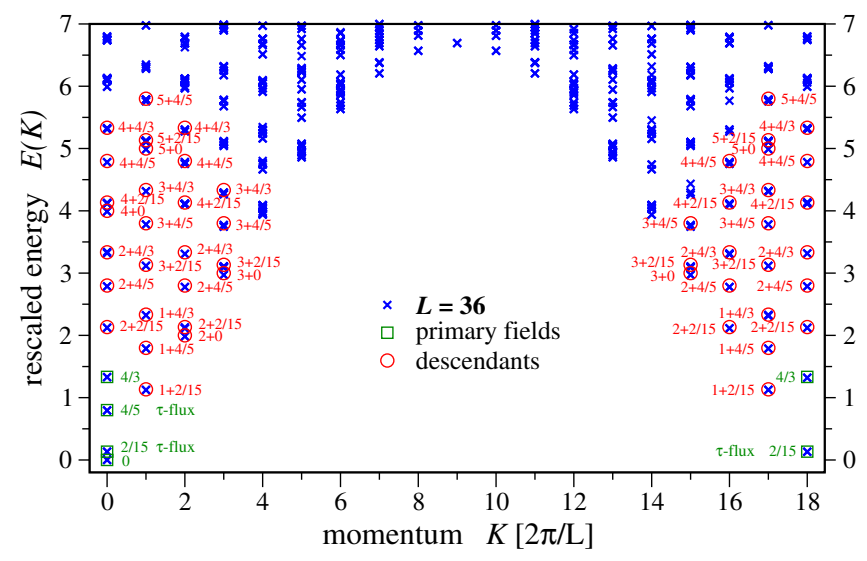

FIG. 3 (color online). Energy spectrum at the $S_{3}$-symmetric point $(\theta \cong 0.176 \pi)$ of the Majumdar-Ghosh chain. The energies have been shifted and rescaled so that the two lowest eigenvalues match the CFT scaling dimensions. The open boxes indicate the positions of the primary fields of the parafermionic subset of the $c=4 / 5$ CFT; fields with topological flux $\tau$ are marked. The open circles give the positions of multiple descendant fields as indicated. they form the order parameter of a critical 3-state Potts model with $S_{3}$ symmetry.

In the case of FM three-particle interaction $J_{3}<0$ the transition at $\theta \cong-0.472 \pi$ between tricritical Ising and gapped phases is described in terms of CFT by the full $c=$ 4/5 minimal model representing [13] the tetracritical Ising model. Again we have unambiguously identified the CFT description of this critical endpoint by assigning the lowenergy states in the energy spectrum similar to Fig. 3 (shown in the auxiliary material [14]). In particular, the topological symmetry forces the system onto the integrable renormalization group trajectory [15] flowing into the gapped phase or the tricritical Ising fixed point, driven again by the relevant operator with dimension $4 / 3$. This operator belongs to the series of least relevant Ising $Z_{2}$-symmetric operators of scaling dimension $2(k+$ $1) /(k+3)$ in the family of multicritical $Z_{2}$ GinzburgLandau theories described by the conformal miminal models [16] ( $k=3$ for tetracritical Ising). The limit $k \rightarrow \infty$ corresponds to the ordinary $\mathrm{SU}(2)$ spin- $1 / 2$ chain, and in this limit this operator becomes the marginal operator driving the transition into the spontaneously dimerized phase [17]. In analogy to the ordinary $S U(2)$ spin- $1 / 2$ chain, at our tetracritical Ising transition into the gapped phase translational symmetry is spontaneously broken; in our case, however, spontaneous breaking of the topological symmetry occurs in addition. As a consequence, we observe a four-fold ground-state degeneracy throughout this gapped phase for chains with even length. The nature of this gapped phase is best characterized at the point $\theta=$ $3 \pi / 2$ that is the anyonic analog of the Majumdar-Ghosh point of the spin-1/2 Heisenberg chain. At this point the four ground states for even $L$ take the exact form

$$
\begin{aligned}
\left|\psi_{\text {no-flux }}\right\rangle= & \left|\tau_{x} \tau \tau_{x} \tau \tau_{x} \tau \ldots\right\rangle+\phi^{-1}|\tau 1 \tau 1 \tau 1 \ldots\rangle \\
& \pm\left[\left|\tau \tau_{x} \tau \tau_{x} \tau \tau_{x} \ldots\right\rangle+\phi^{-1}|1 \tau 1 \tau 1 \tau \ldots\rangle\right] \\
\left|\psi_{\tau-\text { flux }}\right\rangle= & \phi^{-1}\left|\tau_{x} \tau \tau_{x} \tau \tau_{x} \tau \ldots\right\rangle-|\tau 1 \tau 1 \tau 1 \ldots\rangle \\
& \pm\left[\phi^{-1}\left|\tau \tau_{x} \tau \tau_{x} \tau \tau_{x} \ldots\right\rangle-|1 \tau 1 \tau 1 \tau \ldots\rangle\right]
\end{aligned}
$$

where $\tau_{x}=\phi^{-1}|1\rangle+\phi^{-1 / 2}|\tau\rangle$ denotes a normalized superposition of the states $|1\rangle$ and $|\tau\rangle$ on a single site. Note these ground states have total momenta $K=0$ or $K=\pi$, indicating the two-sublattice ordering. There are two states at each momentum, one with a $\tau$-flux and the other without. Of course, we can instead make the simpler linear combinations of these ground states that explicitly break both the topological and sublattice symmetries: these four states are $\left|\tau_{x} \tau \tau_{x} \tau \tau_{x} \tau \ldots\right\rangle,|\tau 1 \tau 1 \tau 1 \ldots\rangle$ and the equivalent states under translation by one site. Note that the density of 1 's [which for these states is $1 /\left(2 \phi^{2}\right)$ and $1 / 2$, respectively] is a simple order parameter that reflects the topological symmetry breaking.

The low-energy excitations in the gapped phase around the MG point are domain walls between the two-sublatticeordered ground states with a low density of 1's - similar to 


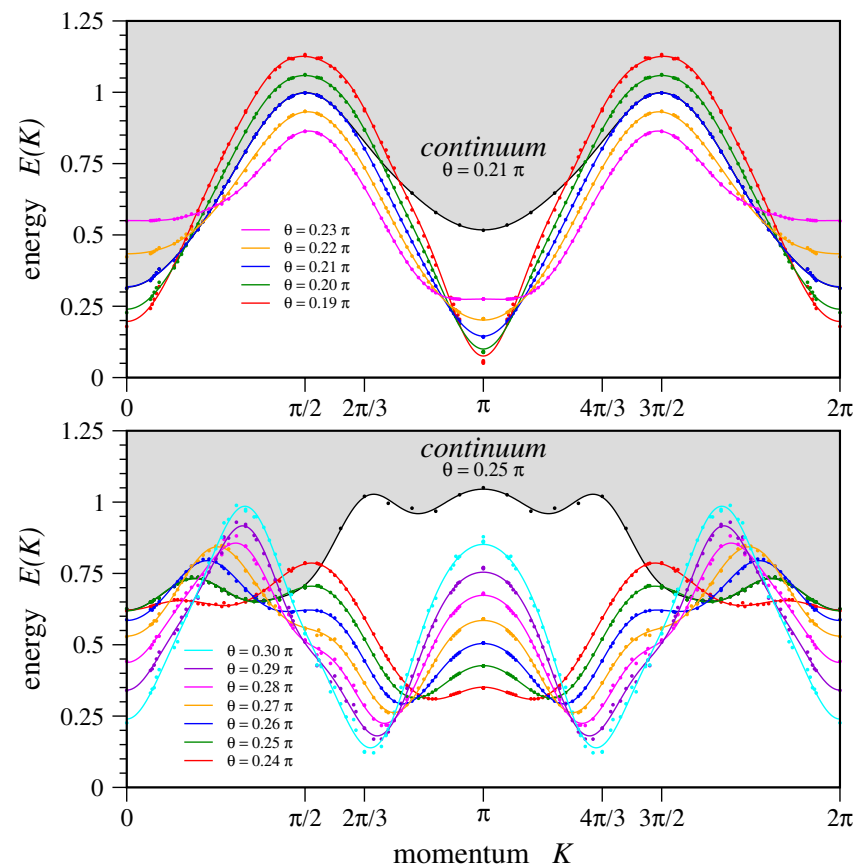

FIG. 4 (color online). Schematic energy spectra in the gapped phase for $0.19 \leq \theta / \pi \leq 0.30$. Below a continuum of scattering states (gray shaded) a quasiparticle band forms. The plot combines data with $24 \leq L \leq 36$. Solid lines are a guide to the eye.

spinon states in the spin-1/2 MG chain. For chains of odd $L$ the periodic boundary conditions force the presence of an odd number of such spinons or walls, and indeed we observe a distinct single-spinon dispersion relation over part of the Brillouin zone as shown in the auxiliary material [14]. Explicitly evaluating the topological symmetry operator [8] we find that these one-spinon states all have a $\tau$-flux. For even length rings we observe a continuum of two-spinon scattering states in the spectrum (shown in the auxiliary material [14]).

At the $S_{3}$-symmetric transition out of the tricritical Ising phase $(\theta \cong 0.176 \pi)$ the system spontaneously breaks only the topological symmetry resulting in a twofold degenerate ground state at total momentum $K=0$ in the gapped phase. With increasing coupling $J_{3}$, away from the transition, an energy gap opens in the spectrum, with a distinct quasiparticle dispersion forming below a continuum of scattering states as shown in Fig. 4. Explicitly evaluating the topological symmetry operator [8] we find that these quasiparticle states all have a $\tau$-flux. For small $J_{3}$, the lowest energy quasiparticle remains at momentum $K=$ $\pi$, with both the gap and the mass increasing with increasing $J_{3}$. Near $\theta \cong 0.24 \pi$ the gap reaches a maximum value and the mass diverges. The minimum of the quasiparticle dispersion bifurcates and continuously moves away from $K=\pi$; see the lower panel in Fig. 4. Eventually, the two minima approach the commensurate momenta $K=$
$2 \pi / 3,4 \pi / 3$ and as these modes soften the system enters the 3 -state Potts critical phase at $\theta \cong 0.316 \pi$.

Finally, we note that when both interaction terms are FM the critical 3-state Potts phase gives way to a small sliver of an incommensurate phase and then a phase with $Z_{4}$ sublattice symmetry. All of these phases appear to be critical or nearly critical. In the incommensurate phase, correlations in the local density of 1's oscillate with a spatial period varying between 3 and 4 lattice spacings.

In conclusion, the anyonic quantum chains possess a topological symmetry that forces their corresponding 2D classical models onto a highly fine-tuned submanifold of their respective phase diagrams. The competition of anyonic exchange interactions allows one to move within this manifold containing a plethora of both, (multi)critical phases and various gapped phases with spontaneously broken topological symmetry.

We acknowledge insightful discussions with N. Bonesteel, M. Freedman, A. Kitaev, M. Levin, and Z. Wang. Our numerical simulations were based on the ALPS libraries [18]. D. A.H. thanks NSF Nos. DMR0213706 and PHY-0551164 for support. A.W.W. L. was supported, in part, by NSF No. DMR-0706140.

[1] A. Kitaev, Ann. Phys. (Leipzig) 303, 2 (2003).

[2] C. Nayak et al., arXiv:0707.1889 [Rev. Mod. Phys. (to be published)].

[3] J. B. Miller et al., Nature Phys. 3, 561 (2007).

[4] M. Dolev et al., Nature (London) 452, 829 (2008).

[5] I. R. Radu et al., Science 320, 899 (2008).

[6] G. Moore and N. Read, Nucl. Phys. B360, 362 (1991).

[7] N. Read and E. Rezayi, Phys. Rev. B 59, 8084 (1999).

[8] A. Feiguin et al., Phys. Rev. Lett. 98, 160409 (2007).

[9] J. S. Xia et al., Phys. Rev. Lett. 93, 176809 (2004).

[10] As for $S U(2)$ spin-1/2 chains the 3 -anyon interaction is a sum of nearest and next-nearest-neighbor interactions.

[11] D. A. Huse, Phys. Rev. Lett. 49, 1121 (1982); Phys. Rev. B 30, 3908 (1984).

[12] C. K. Majumdar and D. K. Ghosh, J. Math. Phys. (N.Y.) 10, 1399 (1969).

[13] J. L. Cardy, Nucl. Phys. B270, 186 (1986).

[14] See EPAPS Document No. E-PRLTAO-101-029829. For more information on EPAPS, see http://www.aip.org/ pubservs/epaps.html.

[15] A. W. W. Ludwig and J. L. Cardy, Nucl. Phys. B285, 687 (1987); A. B. Zamolodchikov, Sov. J. Nucl. Phys. 46, 1090 (1987); A. B. Zamolodchikov, in Integrable Systems in Quantum field Theory and Statistical Mechanics, edited by M. Jimbo, T. Miwa, and A. Tsuchiya (Academic Press, Tokyo, 1989).

[16] A. A. Belavin, A. M. Polyakov, and A. B. Zamolodchikov, Nucl. Phys. B241, 333 (1984).

[17] F. D. M. Haldane, Phys. Rev. B 25, 4925(R) (1982); S. R. White and I. Affleck, ibid. 54, 9862 (1996).

[18] A. F. Albuquerque et al., J. Magn. Magn. Mater. 310, 1187 (2007). 